\title{
OBSERVATION OF COUPLED QUASI-TWO-DIMENSIONAL ELECTRONIC EXCITATIONS IN TILTED MAGNETIC FIELDS
}

\author{
R. BORROFF, R. MERLIN \\ Department of Physics, The University of Michigan, Ann Arbor, MI 48109.1120. USA
}

R.L. GREENE

Department of Physics, University of New Orleans, New Orleans, LA 70148, USA

and

\section{J. COMAS}

Naval Research Laboratory, Washington, DC 20375, USA

Received 1 June 1987; accepted for publication 8 September 1987

We report on a magneto-Raman scattering investigation of free and donor-bound electrons in $\mathrm{GaAs} / \mathrm{Al}, \mathrm{Ga} \mathrm{a}_{\text {- }}$. As quantum wells. For fields perpendicular to the layers, the spectra show intersutband transitions of photcexcited electrons and $1 s-1 s^{\prime}$ donor excitations. Tilted fields iead to subband-Landau level and $: s^{\prime}-2 p^{+}$coupling. Experimental results for the latier case agree very well with variational calcuations. Data on combined intersubband-cyciotron resonances at arbitrary tilt angles are accurately described by expressions valid foi parabolic wells. The parabolic approach is shown to provide a good approximation in situations where coupling to higher subbands can be neglected.

In quasi-two-dimensional (2D) electron systems the motions in the confinement plane $(x, y)$ and perpendicular to it are coupled for magnetic fields $B$ at angles $\theta \neq 0^{\circ}$ with respect to $z[1,2]$. This leads to excitations of mixed character, such as combined intersubband-cyclotron resonances, exhibiting the well-known anticrossing behavior near degeneracy [2-7]. Subband-Landau level coupling has been extensively studied for $2 \mathrm{D}$ systems formed at semiconductor heterojunctions [4-7] and Si-accumulation layers [3] using the technique of cyclotron resonance. For small $\theta$ 's these measurements provide a determination of the energies of intersubband transitions which are forbidden at $\theta=0^{\circ}[2,4]$. Small tilt angles were also used in the far-infrared (FIR) experiments of Jarosik et al. [8] to study crossing of donor levels in quantum-well structures (QWS's). In this work, we report on a Raman scattering (RS) investigation of tilted-field-induced mixing in GaAs/ Al, $\mathrm{Ga}_{1}$, As QWS's for both free and donor-bound electron states. Our results 
complement those obtained from FIR experiments [3-8]; transitions that are weak or forbidden in FIR dominate the Raman spectra. For donor excitations, we find good agreement between the experimental results and those of calculations based on a variational approach [9]. The data on free electrons reveal coupling between the cyclotron mode and $\epsilon_{0} \rightarrow \epsilon_{1}$ transitions associated with the ground and first excited subband states. This coupling has been investigated in the range of parameters where persurbation theory applies, and beyond that range. Analytical expressions derived for parabolic wells [10] were found to describe extremely well the latter results, for arbitrary 8 . This unlikely situation is explained by the close similarity between the coupled-mode equations for parabolic and square wells under conditions where the coupling to higher subbands can be ignored.

A QWS grown by molecular beam expitaxy on (001) GaAs was studied. It consisted of thirty uncoupled GaAs wells of thickness $L=460 \AA$, with $125 \AA$ thick $\mathrm{Al}_{0.24} \mathrm{Ga}_{0.76} \mathrm{As}$ barriers. The sample was doped with $\mathrm{Si}$ donors at the centers of the GaAs slabs with a concentration $N_{\mathrm{D}}=5 \times 10^{15} \mathrm{~cm}^{-3}$. The widtn of the donor spike was $\sim L / 3$. Free-electron excitations were investigated on the same QWS. Carriers were generated either by photoexcitation [11] or by thermal ionization of donors. The laser beam used to obtain RS data also served to photopump the sample. Power densities were in the range $P=2-10 \mathrm{~W} \mathrm{~cm}^{-2}$, giving an estimated free-electron density $\rho \approx 10^{9} \mathrm{~cm}^{-2}[12]$. RS experiments were performed at fields $B \leq 7 \mathrm{~T}$ (the maximum field provided by our split-coil superconducting magnet) and at angles $\theta$ between $0^{\circ}$ and $80^{\circ}$. The laser energy $\hbar \omega_{\mathrm{L}}$ was tuned to resonate with the gap derived from the $E_{0}+\Delta_{0}$ gap of GaAs. At this resonance, the scattering involving states from the conduction band is strongly enhanced [13]. Data were recorded in the $z\left(x^{\prime}, x^{\prime}\right) \bar{z}$ and $z\left(x^{\prime}, y^{\prime}\right) \bar{z}$ backscattering configurations, with $x^{\prime}$ and $y^{\prime}$ denoting the $[110]$ and $[1 \overline{1} 0]$ directions and with $z$ normal to the layers. The former geometry allows scattering by charge-density fluctuations while spin-density fluctuations are allowed in the latter [13]. No appreciable differences were found between spectra in the two configurations, indicating that depolarization effects are negligible. This is consistent with our estimate for a small value of $\rho$.

In fig. 1, we show Raman spectra of the structure with $L=460 \AA$ at two different temperatures. Features labeled $D$ are donor-relaied, which is ciear from their rapid quenching with increasing temperature due to impurity-ionization. The strongest line at $88 \mathrm{~cm}^{-1}$ is mostly due to the transition $1 \mathrm{~s} \rightarrow 1 \mathrm{~s}^{\prime}$ ( $1 \mathrm{~s}^{\prime}$ denotes the lowest donor state associated with the first-excited subband), while the weaker structures at 132 and $137 \mathrm{~cm}^{-1}$ are due, respectively, to the $1 \mathrm{~s} \rightarrow 2 \mathrm{~s}$ and $1 \mathrm{~s} \rightarrow 2 \mathrm{p}^{+}$transitions. The assignment of these transitions is based on the comparison with calculations considered below (for $1 \mathrm{~s} \rightarrow 1 \mathrm{~s}^{\prime}$, see also ref. [12]). As opposed to the $1 \mathrm{~s} \rightarrow \mid \mathrm{s}^{\prime}$ and the $R$ S-allowed $1 \mathrm{~s} \rightarrow 2 \mathrm{~s}$ transitions, the $1 \mathrm{~s} \rightarrow 2 \mathrm{p}^{+}$transition cannot be seen for fields perpendicular to the layers $\left(\theta=0^{\circ}\right)$. The $2 \mathrm{p}^{+}$level transform like $\{x, y\}$ and its Raman activity at $\theta \neq 0^{\circ}$ is derived from the coupling with $1 s^{\prime}$, which transforms like $\{z\}$. Strictly speaking, neither the $1 \mathrm{~s} \rightarrow 2 \mathrm{p}^{+}$nor is $\rightarrow 1 \mathrm{~s}^{\prime}$ transitions are allowed at $m=0$ because the states have different parities ( 0 is the scattering wavevector). 


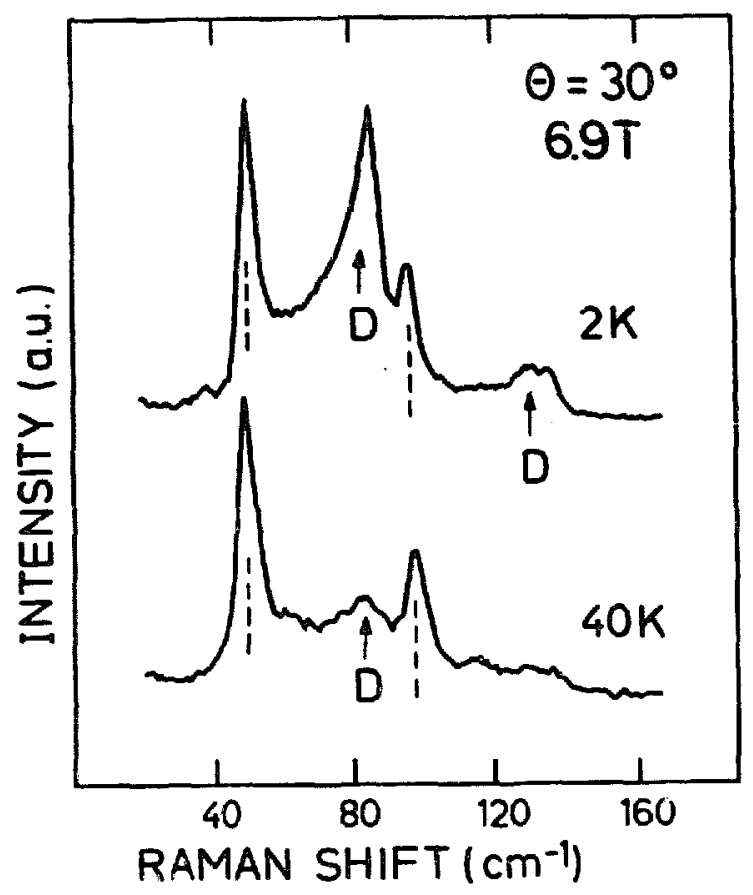

Fig. 1. Raman spectra of the struc11 ie with $L=460 \AA$ showing quenching of donor-related features (D) at high $T$. Dashed lines indicite the positions of the combined intersubband-cyclotron resonances. The scattering geometry $\mathrm{i}^{\prime} z\left(x^{\prime}, y^{\prime}\right) \bar{z}, P \simeq 5 \mathrm{~W} \mathrm{~cm}^{-2}$, and $\hbar \omega_{\mathrm{L}}=1.875 \mathrm{eV} . \theta$ is the angle between the magnetic field and the $z$-axis of the QWS.

However, it is a matter of fact that $\{z\}$-symmetry excitations (e.g., $\epsilon_{0} \rightarrow \epsilon_{1}$ intersubband transitions) can be observed in Raman spectra for $q$ along $z$ [13]. Mechanisms involving $q$-dependent $R S$ are required to account for these observations [13].

The dashed lines in fig. 1 indicate combined intersubband-cyclotron resonances. At $2 \mathrm{~K}$, the intensity of the two peaks (normalized to the intensity of optical phonons) increases roughly linearly with $P$ as expected for scattering due to photoexcited carriers $[11,12]$. The low-energy component at $53 \mathrm{~cm}^{-1}$ is mostly due to $\epsilon_{0} \rightarrow \epsilon$, transitions while the peak at $98 \mathrm{~cm}^{-1}$ derives from the $\Delta n=1$ interLandau level transition. This identification is supported by our results for small angles $\left(\leqslant 10^{\circ}\right)$, where the coupling can be neglected except near degeneracy $[2,4]$. For small angles, the position of the line ascribed to the cyclotron mode closely follows $h \Omega_{\mathrm{c}}=e h \mathrm{~B} / \mathrm{mc}$, with $m=0.07 \mathrm{~m}_{0}\left(\Omega_{\mathrm{c}}\right.$ is the cyclotron frequency and $m_{0}$ is the free electron mass). The nieasured value of $m$ is slightly larger than $m=0.0665 m_{0}$, the mass for electrons at the bottom of the GaAs conduction band. The Raman shift of the line assigned to the $\epsilon_{0} \rightarrow \epsilon_{1}$ transition, at small $\theta$ 's, is $E_{01}=64$ $\mathrm{cm}^{-1}$ (it does not depend much on $|\boldsymbol{B}|$ ). A calculation using the band-gap discontinuities determined by Miller et al. [14] predicts $E_{01}=56 \mathrm{~cm}^{-1}$, which is in reasonably good agreement with the experimental value. It is important to point out that we did not observe a peak at $\hbar \Omega_{c}$ for $B$ normal to the layers. This is unlike 


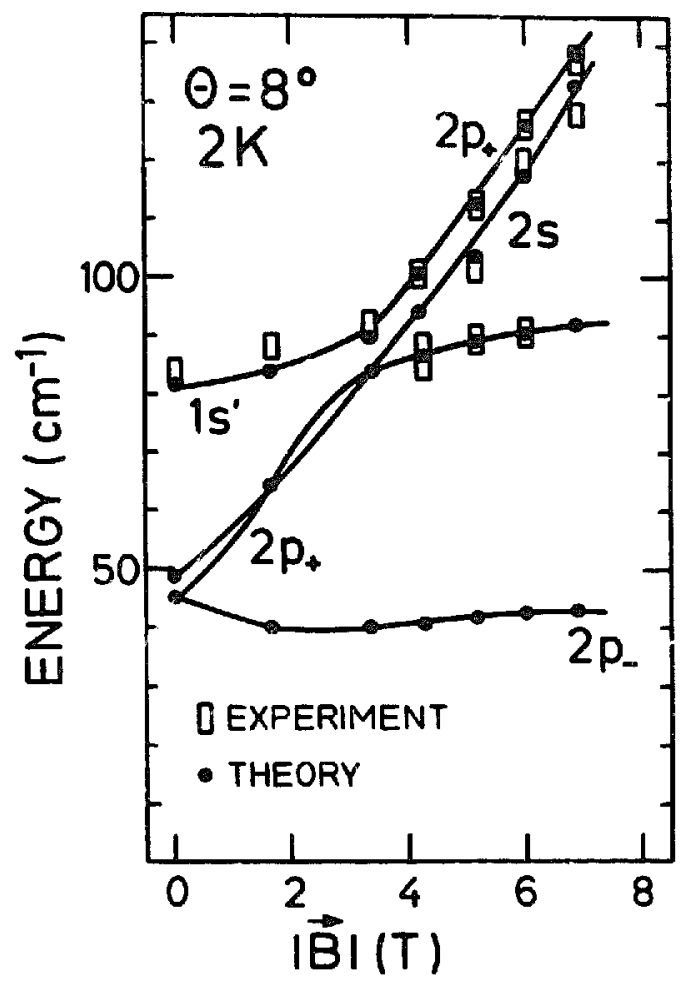

Fig. 2. Comparison between measured and calculated values of $1 s \rightarrow 2 p^{-}, 2 p^{+}, 2 s, 1 s^{\prime}$ transition energies. The theoretical data are for a donor at the center of a $460 \AA \mathrm{GaAs} / \mathrm{Al}_{0.24} \mathrm{Ga}_{0.76} \mathrm{As} \mathrm{QWS}$. The solid lines are a guide to the eye.

modulation-doped QWS's which do show scattering by the cyclotron mode at $\theta=0^{\circ}$ $[15,16]$. We do not know of any reason that might explain this difference.

The positions of donor-related peaks, measured at $\theta=8^{\circ}$, are plotted in fig. 2 as a function of $|B|$. Also shown are results of variational calculations as described in ref. [9], upon which our assignment of the donor transitions is based. The most interesting feature of these data is the coupling between $1 s^{\prime}$ and $2 p^{+}$induced by the tilted field. This is very similar to what has been reported in FIR measurements for donors at the edges of the wells [8].

In fig. 3, we show the measured energies of the coupled intersubband-cyclotron modes for two different tilt angles. Level-repulsion is evident in the figure. $\theta=8^{\circ}$ is within the range where perturbation theory can be applied. The prediction $[2,4]$ of a splitting proportional to $\theta$ for $\hbar \Omega_{\mathrm{c}}=E_{01}$ has been confirmed by our experiments (see the inset). Departures from results of perturbative calculations can be seen in the data of the inset at large angles and in the results for $\theta=60^{\circ}$. In partic$u l a r$, we find that the lower branch of the coupled modes dees not approacin $E_{01}$ for large $B$ 's, but deviates considerably from that value. The theoretical curves in fig. 3 were obtained from expressions valid for parabolic wells [10]. The agreement between theory and experiment is quite remarkable. This is also the case for the asymptotic behavior of the lower branch which is predicted [10] to tend to $E_{0} \cos \theta$ ai high fields. The reason why a square well can be approximated by a 


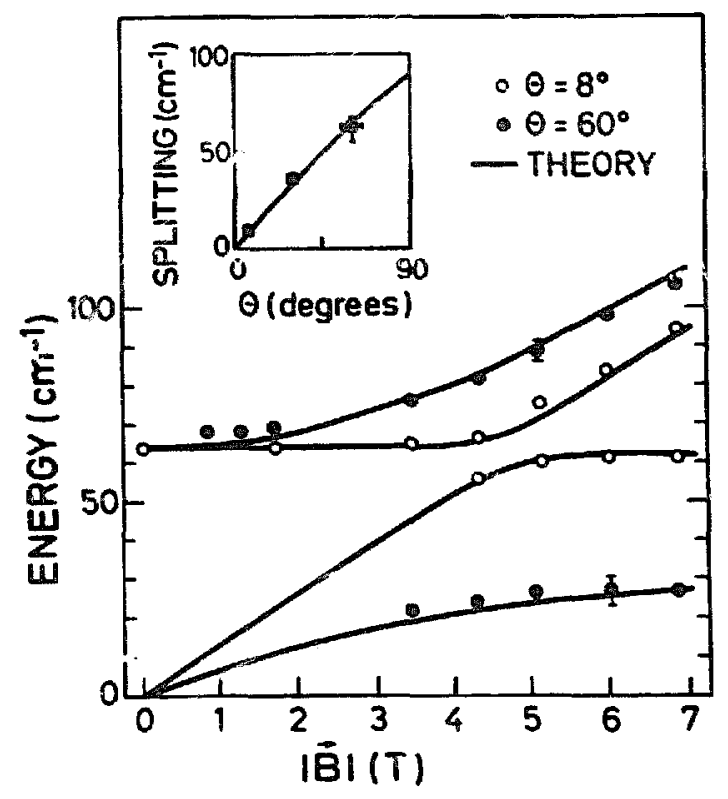

Fig. 3. Energy of the coupled intersubband-Landau level excitations as a function of magnetic field. $\theta$ is the tilt angle. The theoretical curves were obtained from the expressions for parabolic wells [10]. The inset shows coupled-mode splitting versus $\theta$ at a field of $|B|=4.8 \mathrm{~T}$, which corresponds to $\hbar \Omega_{c}=E_{01}$.

parabolic one is basically due to the fact that the corresponding ground and firstexcited subband states are very similar. The matrix elements involved in the coupled-mode problem for the two cases differ by less than $2 \%$. Hence, one can expect that parabolic solutions will apply to square wells if the coupling to higher subbands is not important. A detailed analysis of the coupled equations indicates that this condition is well-fulfilled for the range of parameters in our experiments.

This work was supported by the US Army Research Office under Contract No. DAAG-29-85-K-0175 and the US Office of Naval Research.

\section{References}

[1] F. Stern and W.E. Howard, Phys. Rev. 163 (1967) 816.

[2] T. Ando, Phys. Rev. B19 (1979) 2105; see also, T. Ando, A.B. Fowier and F. Stern, Rev. Mod. Phys. $54(1982) 437$.

[3] W. Beinvogl and J.F. Koch, Phys. Rev. Letters 40 (1978) 1736.

[4]Z. Schlesinger, J.C.M I? ${ }^{\prime}$ ang and S.J. Allen, Phys. Rev. Letters 50 (1983) 2098.

[5] M.A. Brummell, M.A. Hopkins, R.J. Nicholas, I.C. Poral, K.Y. Cheng and A.Y. Cho, J. Phys. C19(1986) L107.

[6] G.L.J.A. Rikken, H. Sigg, C.J.G.M. Langerak, H.W. Myron, J.A.A.J. Perenboom and G. Weimann, Phys. Rev. B34 (1986) 5590

[7] A.D. Wieck, J.C. Maan, U. Merki, J.P. Koithaus, K. Ploog and G. Weimann. Phys. Rev. B35 (1987) 4145 . 
[8] N.C. Jarosik, E. Castano, B.D. McCombe, Y.C. Lee, J. Ralston and G. Wicks, Surface Sci. 170 (1986) 459.

[9] R.L. Greene and K.K. Bajaj, Phys. Rev. B31 (1985) 913.

[10] J.C. Maan, in: Two-Dimensional Systems, Heterostructures, and Superlattices, Eds. G. Bauer, F. Kuchar and H. Heinrich, Springer Series in Solid-State Sciences, Vol. 53 (Springer, Berlin, 1984) p. 183;

R. Merlin, Solid State Commun. 64 (1987) 99.

[11] See: A. Pinczuk, J. Shah, A.C. Gossard and W. Wiegmann, Phys. Rev. Letters 46 (1981) 1341.

[12] T.A. Perry, R. Merlin, B.V. Shanabrook and J. Comas, Phys. Rev. Letters 54 (1985) 2623.

[13] See, e.g., G. Abstreiter, R. Merlin and A. Pinczuk, IEEE J. Quantum Electron. QE-22 (1986) 1771.

[14] R.C. Miller, D.A. Kleinman and A.C. Gossard, Phys. Rev. B29 (1984) 7085.

[15] J.M. Worlock, A. Pinczuk, Z.J. Tien, C.H. Perry, H. Störmer, R. Dingle, A.C. Gossard, W. Wiegmann and R.L. Aggarwal, Solid State Commun. 40 (1981) 867.

[16] A. Pinczuk, D. Heiman, A.C. Gossard and J.H. English, in: Proc. 18th Intern. Conf. on the Physics of Semiconductors, Stockholm, 1987, Ed. O. Engström (World Scientific, Singapore, 1987) p. 557. 